\title{
Reversible Dilation of the Superior Ophthalmic Vein in Intubated Patients
}

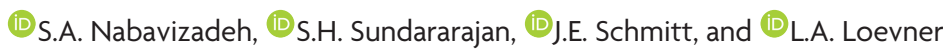

\begin{abstract}
BACKGROUND AND PURPOSE: Superior ophthalmic vein enlargement has typically been associated with increased intracranial or orbital pressure. This study evaluates the incidence of superior ophthalmic vein enlargement in intubated patients without pre-existing intracranial or intraorbital pathologies.
\end{abstract}

\begin{abstract}
MATERIALS AND METHODS: Two cohorts (patients with trauma and epilepsy patients undergoing stereotactic intracranial lead placement) who underwent CT while intubated and shortly following extubation and a cohort of 30 outpatients with a history of headache and normal head CT findings (healthy controls) were included. The superior ophthalmic vein diameter was measured on all scans.

RESULTS: Seventy patients intubated for trauma and 45 patients with intraoperative CT were included $(n=115)$. While intubated, $66 \%$ of the total sample had at least unilateral superior ophthalmic vein dilation of $>2.5 \mathrm{~mm}$ and $48 \%$ had bilateral dilation. Fifty-seven percent of patients with trauma and $84 \%$ of intraoperative patients with dilated superior ophthalmic veins showed reversal of mean superior ophthalmic vein dilation to $<2.5 \mathrm{~mm}$ on postextubation CT. The mean superior ophthalmic vein diameter decreased an average of $1.2 \mathrm{~mm}$ following extubation. Changes in superior ophthalmic vein diameter between intubated and extubated states were statistically significant $(P<.001)$. Differences between the control group and the extubated subjects were not statistically significant $(P=.21)$.
\end{abstract}

CONCLUSIONS: Bilateral dilation of the superior ophthalmic vein is common in intubated patients and usually reverses following extubation. In the appropriate clinical setting, this knowledge will prevent misinterpretation of prominent superior ophthalmic veins as automatically indicative of an underlying pathology.

ABBREVIATION: SOV = superior ophthalmic vein

S uperior ophthalmic veins (SOVs) are routinely detected in high-resolution head CT obtained for a variety of indications. This important orbital venous drainage pathway is located superior to the optic nerve and medially in the anterior part of the orbit, courses between the heads of the superior and lateral rectus muscles, and ultimately exits the orbit via the superior orbital fissure to enter the anterior part of the cavernous sinus. ${ }^{1}$ SOV enlargement is associated with a variety of pathologic entities. For example, lack of intravenous valves contributes to unilateral SOV enlargement in carotid cavernous fistula, Graves orbitopathy, orbital pseudotumor and Tolosa-Hunt syndrome, parasellar and cavernous sinus tumors, orbital varices, arteriovenous malforma-

Received December 16, 2017; accepted after revision April 13, 2018.

From the Department of Neuroradiology, Hospital of the University of Pennsylvania, Philadelphia, Pennsylvania.

Please address correspondence to S. Ali Nabavizadeh, MD, Hospital of the University of Pennsylvania, 3400 Spruce St, Philadelphia, PA 19104; e-mail: seyedali.nabavizadeh@uphs.upenn.edu

http://dx.doi.org/10.3174/ajnr.A5699 tions, orbital hematoma, and other orbital infectious or inflammatory etiologies. ${ }^{2-5}$ Bilateral superior ophthalmic vein enlargement has been reported in patients with diffuse cerebral swelling and increased intracranial pressure. ${ }^{4,6,7}$ Given such associations, SOV enlargement often raises concerns for an underlying pathologic state. However, in our clinical radiology practice, we have empirically observed reversible SOV enlargement in intubated patients (Fig 1). The aim of the current study was to determine the incidence and reversibility of SOV enlargement in 2 cohorts evaluated by CT during and after intubation. We hypothesized that intubation contributes to reversible bilateral enlargement of the SOV.

\section{MATERIALS AND METHODS Patient Population}

The study was performed with approval of the institutional review board and was compliant with guidelines of the Health Insurance Portability and Accountability Act. A radiology information system key word search using MONTAGE Search and Analytics software (Montage Healthcare Solutions, Philadelphia, Pennsylva- 


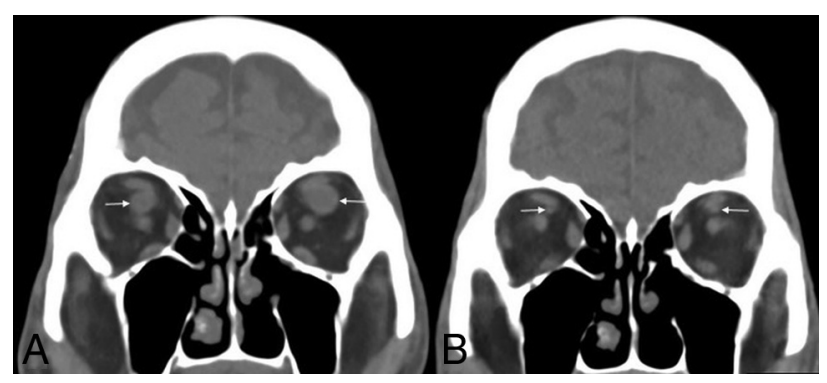

FIG 1. A 75-year-old man presenting to the emergency department as a trauma alert after experiencing a fall. No intracranial hemorrhage or acute intracranial abnormality was found. The patient was intubated for airway protection secondary to aspiration. The right SOV diameter was $7.9 \mathrm{~mm}$ and the left SOV diameter was $10 \mathrm{~mm}$ on initial head CT (A, arrows). On 2-week follow-up CT following extubation, the SOV diameter significantly decreased bilaterally ( $B$, arrows).

nia) was performed on all CT examinations of the brain between July 2010 and July 2016 to find 2 cohorts of intubated patients. The first cohort consisted of patients with epilepsy with intraoperative CT scans for stereotactic placement of subdural or depth electrodes. Search terms included "intraoperative," "depth electrodes," and "subdural electrodes." All intraoperative imaging was acquired during positive pressure ventilation. These patients with epilepsy did not have any intracranial mass effect, and all underwent a follow-up CT within 1 week of the operation to monitor the electrode placement.

The second cohort consisted of emergency department patients with trauma without intracranial hemorrhage or acute intracranial abnormality. Search terms included "fall," "MVC," and "trauma." This included patients who had fallen, were involved in motor vehicle collisions, or other types of trauma. Airway maintenance in this emergency department cohort was established with or without positive pressure ventilation. The included patients also had head CT imaging performed following intubation and within 4 weeks following extubation. The reason for the follow-up CT in patients with trauma ranged from altered mental status to fall or headache. Patients who had any structural intracranial pathology (eg, intracranial hemorrhage, cerebral edema, or mass effect) that would contribute to elevated intracranial or intraorbital pressure were excluded from the study. Finally, a third cohort was included. These included patients who had head CT as a work-up for their headache and normal CT examination findings. Search terms included "headache," "CT," and "normal."

\section{CT and Image Analysis}

All CT was performed without contrast administration. In the intraoperative cohort, the SOV transverse diameter was recorded on coronal reformations of 1-mm-section axial images acquired on our institutional portable CT scanner (CereTom, 8-slice; NeuroLogica, Danvers, Massachusetts). The transverse diameter of the emergency department cohort was recorded on coronal reformations of 1-mm-section axial source images acquired on our institutional emergency department CT scanners (Somatom Definition Edge, 128-slice, and Somatom Definition Flash, 128slice; Siemens, Erlangen, Germany). Dilated SOVs were considered to be $\geq 2.5 \mathrm{~mm}$ based on prior studies of mean SOV diameter ranging from 1.7 to $2.2 \mathrm{~mm}$ with $\mathrm{SD} \pm 1.2 \mathrm{~mm}^{8,9}$ and also another study that demonstrated this threshold is associated with increased intracranial pressure. ${ }^{7}$ SOV diameter was recorded in all patients, posterior to the globe of the eye where the SOV crossed the inferior aspect of superior rectus muscle, by a neuroradiology fellow (S.H.S.). To assess interrater reliability of measurements, a boardcertified neuroradiology attending with 5 years of experience (S.A.N.) measured the SOV diameter in a random subset of 10 subjects from each cohort $(n=20)$.

\section{Statistical Analysis}

Data were imported into the $\mathrm{R}$ statistical environment (Version 3.2.4; http://www.r-project.org/) for analysis. ${ }^{10}$ First, descriptive statistics were calculated. Interrater reliability was assessed via intraclass correlations. Data were then visualized to assess normality. We first tested differences between intubated and extubated states in our intraoperative and trauma groups.

Because the normality assumptions required for parametric statistics were not met in these data, Wilcoxon (paired) signed rank tests were performed to test differences with the intubation state. Left, right, and mean SOV diameters were tested. To ensure that cohort differences (ie, intraoperative versus trauma) did not confound the results, we repeated our analysis with each cohort separately. The effect of the intubation state on the number of subjects with SOV diameters above a threshold of $2.5 \mathrm{~mm}$ was assessed via the McNemar test for the trauma and intraoperative groups separately. The Bonferroni correction on 12 statistical tests was used to adjust for multiple testing.

To provide an alternate baseline for SOV diameter, we also compared our trauma and intraoperative cohorts with a third group consisting of outpatient healthy controls. Three-way Kruskal Wallis tests (outpatient, intraoperative, and trauma) were performed to identify group differences in SOV caliber, with post hoc pair-wise comparisons performed via the Dunn test.

\section{RESULTS}

A total of 145 patients were included in our study (30 patients in the control group, 45 patients in the intraoperative cohort, 70 patients in the emergency department trauma cohort). In the control group, there were 23 women and 7 men (mean age, $54.78 \pm 17.7$ years; range, $24-93$ years). Within the intraoperative cohort, 24 patients were men and 21 patients were women (mean age, $56.1 \pm 16.2$ years; range, $21-84$ years). In the emergency department trauma cohort, 48 patients were men and 22 patients were women (mean age, $58.1 \pm 21.7$ years; range, $21-87$ years).

Initial imaging following intubation showed that 55 of the 115 patients in the experimental cohorts (48\%) demonstrated dilation of the bilateral SOVs to $\geq 2.5 \mathrm{~mm}$. Sixty percent of patients in the intraoperative cohort (27/45) and $40 \%$ of patients in the emergency department trauma cohort (28/70) had bilateral SOV dilation. Sixty-six percent of patients (76/115) demonstrated at least unilateral dilation of the superior ophthalmic veins when intubated. Of the $58 \%(67 / 115)$ of patients with dilated mean SOVs during intubation, $47 / 67$ (70\%) demonstrated reversal of average SOV dilation below $2.5 \mathrm{~mm}$ on postextubation CT. Differences in mean SOV dilation between intubated and extubated 
states were statistically significant for the combined sample cohort $\left(\right.$ McNemar $\left.\chi^{2}=42.18, d f=1, P<.001\right)$ as well as when examining the intraoperative $\left(\chi^{2}=25.03, d f=1, P<.001\right)$ and trauma cohorts $\left(\chi^{2}=15.43, d f=1, P<.001\right)$ separately.

SOV diameter decreased for most subjects following extubation (Fig 2); for example, mean SOV diameter decreased in $88 \%$ of subjects (102/115). When considering group means, SOV caliber decreases following extubation ranged from 0.90 to $1.14 \mathrm{~mm}$ (Table). Reductions in mean, left, and right SOV diameters were all statistically significant $(P<.001)$. These differences remained significant when examining the trauma and intraoperative subgroups separately $(P<.001)$. The intraclass correlation for interrater reliability was 0.93 .
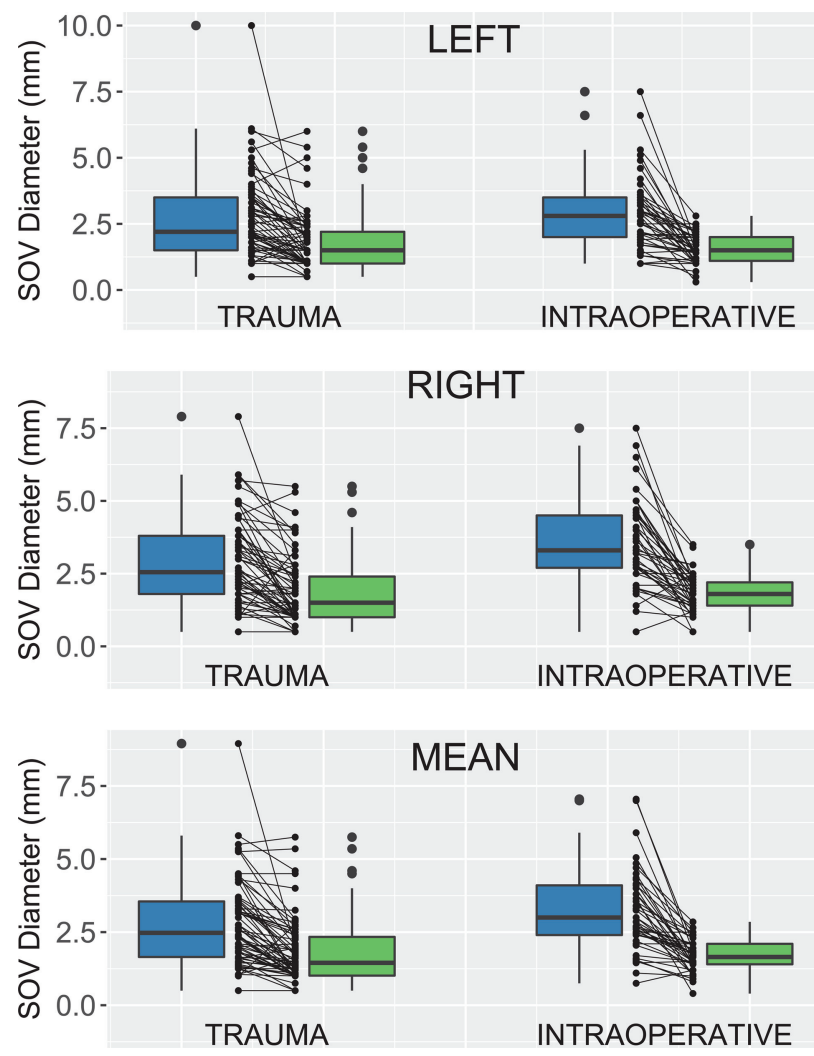

FIG 2. SOV diameter during and after intubation for both emergency department trauma and intraoperative cohorts. Both paired boxplots and dotplots are provided. In each cohort, boxplots on the left represent patients while intubated, with boxplots on the right showing SOV values following extubation. Left, right, and mean (average) SOV diameters are shown separately.

Differences in SOV caliber before and after intubation

\begin{tabular}{|c|c|c|c|c|c|}
\hline \multirow[b]{2}{*}{ Cohort (No.) } & \multirow[b]{2}{*}{ sov } & \multicolumn{2}{|c|}{ Mean (SD) } & \multirow{2}{*}{$\begin{array}{c}\text { Mean } \\
\text { Difference } \\
(99 \% \mathrm{Cl})\end{array}$} & \multirow{2}{*}{$\begin{array}{c}P \\
\text { Value }\end{array}$} \\
\hline & & Intubated & Extubated & & \\
\hline \multirow[t]{2}{*}{ Intraoperative (45) } & Right & $3.56(1.51)$ & $1.82(0.66)$ & 1.74 (1.31-2.17) & $<.001^{\mathrm{a}}$ \\
\hline & Left & $2.91(1.41)$ & $1.53(0.54)$ & $1.36(0.95-1.79)$ & $<.001$ \\
\hline \multirow[t]{2}{*}{ Trauma (70) } & Right & $2.88(1.48)$ & $1.86(1.16)$ & $1.02(0.74-1.30)$ & $<.001$ \\
\hline & Left & 2.68 (1.59) & 1.78 (1.11) & $0.90(0.5-1.21)$ & $<.001$ \\
\hline \multirow[t]{2}{*}{ Control (30) } & Right & - & $1.88(0.51)$ & - & $\leq .001^{\mathrm{b}}$ \\
\hline & Left & - & $1.83(0.49)$ & - & $<.001$ \\
\hline
\end{tabular}

${ }^{a}$ Bonferroni-corrected Wilcoxon paired signed rank tests between intubated and extubated states.

b Three-way Kruskal-Wallis test between controls and intubated cohorts. Post hoc pair-wise comparisons between controls and the experimental groups were also statistically significant $(P<.001)$. Comparisons with extubated patients were not statistically significant.
Mean SOV diameter in the outpatient control group was $1.86 \pm 0.46 \mathrm{~mm}$. SOV diameter in the controls was comparable with that in the extubated experimental cohorts (Table), with only $3 / 30(10 \%)$ having a mean SOV of $>2.5 \mathrm{~mm}$. Differences between the control group and the extubated subjects were not statistically significant for left $(P=.11)$, right $(P=.30)$, or average SOV diameter $(P=.21)$. However, there were statistically significant group differences between mean SOV diameters in controls and the intubated cohorts (Kruskal-Wallis $\chi^{2}=22.3121, d f=2$, $P<.001)$. Post hoc comparisons demonstrated that the mean SOV diameter in the control group was significantly lower than that in both intubated trauma $(z=3.27, P=.005)$ and intraoperative $(z=4.72, P<.001)$ cohorts.

\section{DISCUSSION}

The current study provides evidence of a strong association between intubation state and SOV diameter in 2 different groups of patients without intracranial mass or increased intracranial pressure. The result of our study is concordant with prior cerebral angiography studies that have shown that the SOV is more frequently visualized in intubated patients. ${ }^{11-13}$ Ricker et al ${ }^{12}$ demonstrated that the SOV was visualized on $43 \%$ of cerebral angiograms obtained for intubated patients compared with only $22 \%$ on nonintubated patients. In a study of 452 patients, Servo ${ }^{14}$ showed that intubation increased the visualization of the SOV. However, this finding was only observed in patients with normal flow direction from the facial vein to the cavernous sinus, but not in patients with reversed flow. In addition, Servo showed statistically significant differences on SOV visualization based on middle cerebral and uncal vein drainage either normally draining into the cavernous sinus or bypassing direct drainage via normal-variant collateral pathways. ${ }^{14}$

The effect of the direction of flow in the facial vein has also been studied using 3D TOF MR angiography. ${ }^{15}$ Besides the effect of anatomic variations in the venous system, another proposed mechanism for increased visualization of the SOV in intubated patients in cerebral angiography is facilitated accumulation of contrast in the nasal mucosa. ${ }^{1,13}$ The above angiographic studies have been performed in patients with intracranial pathologies ranging from intracranial mass lesions and hydrocephalus to other pathologies that could increase the intracranial pressure. In addition, the diameter of the SOV was not measured in these studies. Our study is the first of its kind to investigate the effect of intubation on SOV using cross-sectional imaging in patients without intracranial mass or increased intracranial pressure, to our knowledge.

Our findings suggest that bilateral SOV dilation is common in intubated patients and that it is usually reversible following extubation. All patients in the intraoperative cohort received positive pressure ventilation (compared with the emergency department trauma cohort in which patients may or may not have received positive pressure ventilation). Positive pressure ventilation intubation might contribute to significantly 
larger and more frequent changes in SOV diameter in this cohort, perhaps because of accentuated intrathoracic pressure mediating venous backflow into the head and neck. In addition, there are other factors that could contribute to changes in SOV diameter such as the state of hydration and blood pressure at the time of imaging and the effect of sedative-anesthetic medications. Nevertheless, the purpose of this study was not necessarily to surmise the mechanism by which this process occurs. Rather, it was to demonstrate that with intubation, it is certainly possible for prominence of the SOVs to be attributable to intubation status rather than immediately indicative of an underlying abnormality. In the appropriate clinical setting, awareness of this entity may help prevent misinterpretation of prominent SOVs as automatically indicative of an underlying pathology.

Another observation is that the diameter of the SOV in some patients in both the intraoperative and trauma cohorts stayed the same or even slightly increased. We believe that this finding could be due to dilated SOVs as a normal variation as suggested by prior studies ${ }^{8,9}$ or it could be the result of additional factors that were not measured in this retrospective study, such as the state of hydration and blood pressure at the time of imaging and the effect of sedative-anesthetic medications.

This study has some limitations. The lack of CT-evident intracranial or intraorbital findings was used as the criteria for delineating lack of elevated intracranial and intraorbital pressure, respectively. The standard for measurement of elevated intracranial pressure-lumbar puncture or intraventricular catheter resultswas not logistically feasible for this study. Additionally, although it was known that positive pressure ventilation was used in all intraoperative cases and intermittently in our emergency department trauma cohort, exact numeric data regarding pressure settings were not available in the medical record because such data are not frequently recorded by the anesthesiologists during their routine documentation. In addition, given the retrospective nature of the study, reliable data about the state of hydration and blood pressure at the time of imaging could not be obtained. Finally, dedicated vascular imaging was not generally available; therefore, it was not possible to correlate our findings with vascular anatomy, including venous anatomic variations in the face, skull base, and brain. Future prospective studies could incorporate some of this information to better understand the causes of SOV dilation.

\section{CONCLUSIONS}

Bilateral dilation of the SOV is common in intubated patients and usually reverses following extubation. In the appropriate clinical setting, knowledge of this entity will prevent misinterpretation of prominent SOVs as automatically indicative of an underlying pathology.

\section{REFERENCES}

1. Rhoton AL. Cranial Anatomy and Surgical Approaches. Philadelphia: Lippincott Williams \& Wilkins; 2003

2. Wei R, Cai J, Ma X, et al. Imaging diagnosis of enlarged superior ophthalmic vein. Zhonghua Yan Ke Za Zhi 2002;38:402-04 Medline

3. Peyster RG, Savino PJ, Hoover ED, et al. Differential diagnosis of the enlarged superior ophthalmic vein. J Comput Assist Tomogr 1984;8: 103-07 CrossRef Medline

4. Servo A, Jääskinen J. The superior ophthalmic vein and tumours of the sellar area. Acta Neurochir (Wien) 1983;68:195-202 CrossRef Medline

5. Adam CR, Shields CL, Gutman J, et al. Dilated superior ophthalmic vein: clinical and radiographic features of 113 cases. Ophthalmic Plast Reconstr Surg 2018;34:68-73 CrossRef Medline

6. Khanna RK, Pham CJ, Malik GM, et al. Bilateral superior ophthalmic vein enlargement associated with diffuse cerebral swelling: report of 11 cases. J Neurosurg 1997;86:893-97 CrossRef Medline

7. Lirng JF, Fuh JL, Wu ZA, et al. Diameter of the superior ophthalmic vein in relation to intracranial pressure. AJNR Am J Neuroradiol 2003;24:700-03 Medline

8. Reis CV, Gonzalez FL, Zabramski JM, et al. Anatomy of the superior ophthalmic vein approach for direct endovascular access to vascular lesions of the orbit and cavernous sinus. Neurosurgery 2009;64(5 Suppl 2):318-23; discussion 323 CrossRef Medline

9. Tsutsumi $S$, Nakamura $M$, Tabuchi T, et al. The superior ophthalmic vein: delineation with high-resolution magnetic resonance imaging. Surg Radiol Anat 2015;37:75-80 CrossRef Medline

10. R Core Team. The R Project for Statistical Computing. https://www. R-project.org/. Accessed November 1, 2017

11. Hacker H, Porrero M. Demonstration and significance of the ophthalmic vein in carotid angiography [in German]. Fortschr Geb Rontgenstr Nuklearmed 1969;110:656-63 CrossRef Medline

12. Ricker $H$, Reiner I, Nadjmi M. Significance of the superior ophthalmic vein in the phlebogram [in German]. Radiologe 1972;12:414-16 Medline

13. Bradac GB, Simon RS, Leonhardt W. The ophthalmic vein in the carotid angiogram. Neuroradiology 1974;8:39-47 CrossRef Medline

14. Servo A. Visualization of the superior ophthalmic vein on carotid angiography. Neuroradiology 1982;23:141-6 Medline

15. Uchino A, Egashira R, Nomiyama K, et al. Visualization of the superior ophthalmic veins by 3 Tesla 3D-TOF-MR angiography. Neuroradiol J 2008;21:619-22 CrossRef Medline 